\title{
Probabilistic Detection of Mobile Targets in Heterogeneous Sensor Networks
}

\author{
Loukas Lazos \\ Network Security Lab \\ Dept. of Electrical Engineering \\ University of Washington \\ llazos@u.washington.edu
}

\author{
Radha Poovendran \\ Network Security Lab \\ Dept. of Electrical Engineering \\ University of Washington \\ rp3@u.washington.edu
}

\author{
James A. Ritcey \\ Dept. of Electrical Engineering \\ University of Washington \\ jar7@u.washington.edu
}

\begin{abstract}
Target detection and field surveillance are among the most prominent applications of Sensor Networks (SN). The quality of detection achieved by a $\mathrm{SN}$ can be quantified by evaluating the probability of detecting a mobile target crossing a Field of Interest $(F o I)$. In this paper, we analytically evaluate the detection probability of mobile targets when $N$ sensors are stochastically deployed to monitor a $F o I$. We map the target detection problem to a line-set intersection problem and derive analytical formulas using tools from Integral Geometry and Geometric Probability. We show that the detection probability depends on the length of the perimeters of the sensing areas of the sensors and not their shape. Hence, compared to prior work, our formulation allows us to consider a heterogeneous sensing model, where each sensor can have a different and arbitrary sensing area. We also evaluate the mean free path until a target is first detected, a measure of timely detection. We verify our theoretic derivations via simulations.
\end{abstract}

\section{INTRODUCTION}

Target detection is one of the fundamental services provided by most Sensor Networks (SN). For the purposes of target detection, a number of sensors $N$ are deployed to monitor a Field of Interest $F o I$. The sensor deployment can be either stochastic or deterministic depending on the application and the FoI. Stochastic deployment is preferred when the $F_{O} I$ is not under the designer's control at the time of deployment (hostile environment), or when it is more cost-effective to randomly deploy the sensors than systematically place them (large-scale networks) [3], [10], [13].

Once the SN is deployed, targets are detected using one or more sensing modalities such as optical, mechanical, acoustic, thermal, RF and magnetic sensing. In fact, to ensure robustness and enhance performance, oftentimes a sensor fusion approach is required [1]. As an example, a surveillance system can be realized via fusion of data aggregated from various sensor modalities: infrared, $\mathrm{CCD}$, pressure and acoustic sensors.

Depending on the modality, sensing areas have any arbitrary shape, a reality significantly different from the widely adopted idealized unit disk model [2]-[6]. Moreover, in sensor fusion scenarios, devices of different modalities have heterogeneous sensing capabilities. To date, the target detection problem under a heterogeneous sensing model has not been studied, since prior work assumes identical sensing areas for all sensors [2]-[6], [9]-[12]. In this paper, we address the problem of quantifying the target detection capability of stochastically deployed SN, when sensors have heterogeneous sensing capabilities.

In stochastically deployed networks, the target detection is achieved only probabilistically. One metric that quantifies the detection capability of a SN, is the probability of target detection by at least one sensor [3]. This metric provides a worst-case guarantee on the number of sensors able to detect a mobile target. For applications that require enhanced fault tolerance and reduced false alarms, detection by more than one sensors is critical [9], [10], [13]. In such cases, the quality of detection can be quantified by evaluating the probability of detection by at least $k$ sensors, where $k$ is a design parameter. Furthermore, in several applications it is critical that the target is detected in a timely fashion. The relevant metric that quantifies the timeliness of the detection, is the mean free path until the first detection. Given a target velocity model, the mean free path can be related to the mean time until detection [2], [3].

For target detection, we consider two detection models. In the first model called the Instant Detection model (ID), a sensor $s$ detects a target $X$ when the trajectory of $X$ intersects the sensing area of $s$. A similar model has also been considered in [2]-[6]. However, in more realistic scenarios, a sensor needs to collect multiple samples of the target before it can perform reliable detection [7], [8]. Hence, we also consider the Sampling Detection model (SD), where a sensor $s$ must sample the target $X$ for at least $t_{t h}$ units of time, before $s$ can reliably determine the presence of $X$. Several previous works have assumed the Energy Detection model (ED), where a target is detected if the energy level measured by a set of sensors exceeds a pre-defined threshold [9]-[12]. The ED model also requires a threshold number of samples to detect a target [8], and hence, ED is a special case of the SD model.

\section{A. Our Contributions}

In this paper we make the following contributions. We map the target detection problem to a line-set intersection problem. Based on our mapping, we use tools from Integral Geometry and Geometric Probability to analytically evaluate the probability of detecting targets moving at a random direction within the $F_{O I}$. 


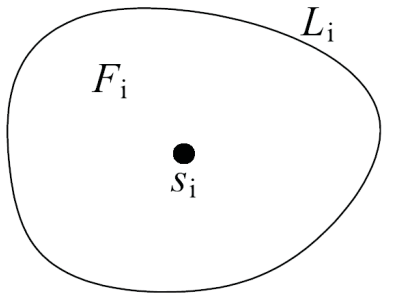

(a)

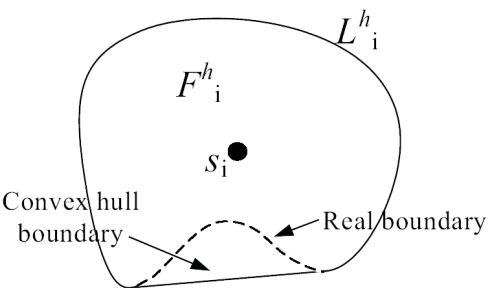

(b)

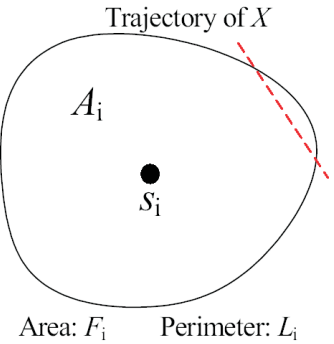

(c)

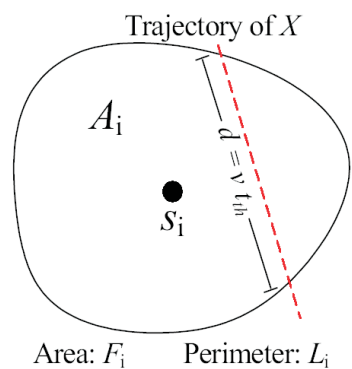

(d)

Fig. 1. (a) A convex sensing area $\mathcal{A}_{i}$ of size $F_{i}$ and perimeter $L_{i}$, (b) a non-convex sensing area with a convex hull boundary of size $L_{i}^{h}$ and area size $F_{i}^{h}$, (c) the instant detection model: a target $X$ is detected if its trajectory crosses the sensing area of $s_{i}$, (d) the sampling detection model: a target $X$ is detected if it is sensed for at least $t_{t h}$ units of time. Given a constant speed $v$ of $X$, the length of the trajectory of $X$ within the sensing area of $s_{i}$ must be greater than $v t_{t h}$.

Our formulation shows that the target detection probability is independent of the shape of the sensing areas of the devices, and depends only on the length of the perimeters of the sensing areas (length of the perimeters of the convex hulls of the sensing areas, for non-convex shapes). Hence, compared to prior work, [2]-[6], [9]-[12], our mapping allows us to consider a heterogeneous sensing model where, even sensors with the same sensor modality need not have identical sensing capabilities. Using our formulation, we analytically evaluate the target detection probability for heterogeneous SN, and derive the results for homogeneous SN, as a special case.

We study the problem of target detection under both the ID and SD models, and show that the target detection problem under the SD model can be reduced to the target detection problem under the ID model, by introducing the concept of the effective sensing area. We also evaluate the mean free path until the target is first detected, a critical measure for timely detection. Our derivations provide an analytic tool for network designers to select parameters such as the number of sensors, and type of sensing areas (perimeter lengths) that guarantee a minimum target detection probability.

The rest of the paper is organized as follows. In Section II we present related work. In Section III, we state our model assumptions, formulate the target detection problem, and provide relevant background. In Section IV, we analytically evaluate the target detection probability and the mean free path until the first detection. In Section V, we verify our theoretical results via simulations. In Section VI, we present our conclusions.

\section{RELATED WORK}

The target detection problem in $\mathrm{SN}$ has been a topic of study under different metrics and assumptions [2]-[6], [9], [11]-[13]. In [2], the authors investigate the tradeoff between detection quality and power conservation. In [3], analytic formulae for the mean delay until a target is detected are provided, when targets move on a straight line at a constant speed. In [9], [11], [12], the authors proposed a collaborative detection model, where sensors collectively arrive at a consensus about the presence of a target. Their formulation assumes that the detection capability of each sensor decays as a function of distance. In [21], the authors provided algorithms for optimum k-coverage of the boundary of a $F o I$ that yields target detection with certainty. All prior work mentioned above [2], [3], [9], [11]-[13], assume that sensors have homogeneous sensing capabilities conforming to the idealized unit disk model.

\section{Model Assumptions, Problem Formulation And BACKGROUND}

\section{A. Network and Target Model Assumptions}

In this section we state our assumptions about the network deployment, as well as the sensor and target models we adopt.

Sensor Deployment: We assume that $N$ sensors are identically and independently distributed within a planar $F o I$, $\mathcal{A}_{0}$, according to a random (uniform) distribution. The $F_{O} I$ is a connected and closed set of perimeter $L_{0}$ of arbitrary shape. In the case where the $F_{O} I$ is not convex, we assume that the perimeter, denoted as $L_{0}^{h}$, of the convex hull of $F o I$ is known.

Target Model: We assume that the trajectories of the mobile targets are straight lines, with all trajectories crossing the $\mathrm{FoI}$ being equiprobable. Although such an assumption constraints the space of all possible trajectories, we adopt it for two reasons.

(a) Given any arbitrary entry and exit point in the FoI, moving on a straight line minimizes the length of the trajectory of the target within the FoI (minimizes the time that the target can be detected). Hence, the target detection probability assuming line trajectories is the worst case probability compared to the detection of any other possible trajectory. The worst case analysis allows us to compute network parameters such as sensor density and length of the perimeters of the sensing areas, so that target detection is guaranteed with a minimum probability.

(b) If an arbitrary trajectory is considered, the parameters of the trajectory (length, curvature, multiple self-crossing points) need to be specified in the model, in order to analytically evaluate the probability of target detection. On the other hand, line trajectories have a simple parameterization that facilitates the analytical calculation and physical interpretation. 
Though we do not present it in this paper, our formulation can be extended to include trajectories of arbitrary shapes with known parameters, as well as three dimensional FoI. Furthermore, straight line motion models have also been assumed in previous works addressing the target detection problem [2], [3].

Sensing Model: We assume that each sensor $s_{i}, i=1 \ldots N$ has a sensing area $A_{i}$ that is a closed and connected set of perimeter $L_{i}$. In the case where the sensing area is not convex, we assume that the perimeter, denoted as $L_{i}^{h}$ of the convex hull of $A_{i}$ is known. Based on our assumptions, sensors need not have an identical sensing area $A_{i}$. Figure 1 (a) illustrates a sensing area $\mathcal{A}_{i}$ of convex shape. Figure 1(b) illustrates a nonconvex sensing area and the equivalent convex hull boundary. For detecting a mobile target $X$ we consider the following two cases:

(a) ID model: A target $X$ is detected by a sensor $s_{i}$ if the trajectory of $X$ crosses the sensing area of $s_{i}$.

(b) SD model: A target $X$ is detected by a sensor $s_{i}$ if $X$ is sensed (sampled) for at least $t \geq t_{t h}$ units of time, where $t_{t h}$ is a design parameter.

Figure 1(c) illustrates detection based on the ID model which places no constraint on the length of the line segment of the trajectory within $\mathcal{A}_{i}$. Figure $1(\mathrm{~d})$ illustrates detection based on the SD model, where a target $X$ moving at a constant speed $v$ is detected, only if the trajectory inside $\mathcal{A}_{i}$ is longer than $v t_{t h}$. We now provide our formulation for the moving target detection problem.

\section{B. Problem Formulation}

Mobile target detection problem: Given a FoI $\mathcal{A}_{0}$ of perimeter $L_{0}$ sensed by $N$ sensors with sensor $s_{i}$ having a sensing area $\mathcal{A}_{i}$ of perimeter $L_{i}$, randomly and independently deployed within the FoI, compute the probability $P_{D}(k)$ that a target $X$ randomly crossing $\mathcal{A}_{0}$ is detected by at least $k$ sensors.

Mapping the mobile target detection problem: The problem of mobile target detection under stochastic deployment can be mapped to a line-set intersection problem by performing the following mapping. Let the $F o I$ be mapped to a bounded set $S_{0}$, defined as a collection of points in the plane with perimeter length $L_{0}$. Let the sensing area of sensor $s_{i}$ be mapped to a bounded set $S_{i}$ with perimeter length $L_{i}$. Let the trajectory of the target $X$ be mapped to a straight line $\ell(\xi, \theta)$ in the plane, with parameters $\xi$ and $\theta$ be the shortest distance of $\ell$ to the origin of a coordinate system, and $\theta$ be the angle of the line perpendicular to $\ell$ with respect to the $x$ axis. Then, the mobile target detection problem for stochastic $\mathrm{SN}$ is equivalent to the following line-set intersection problem.

Line-set intersection problem: Given a bounded set $S_{0}$ of perimeter length $L_{0}$ and $N$ sets $S_{i}$ of perimeter length $L_{i}$, randomly and independently placed inside $S_{0}$, compute the probability $P_{D}(k)$ that a random line $\ell$ intersecting $S_{0}$, also
TABLE I

MAPPING THE MOBILE TARGET DETECTION PROBLEM TO THE LINE-SET INTERSECTION PROBLEM

\begin{tabular}{|ccc|}
\hline Mobile Target Detection & $\leftrightarrow$ & Line-Set Intersection \\
\hline Number of sensors $N$ & $\leftrightarrow$ & Number of sets $N$ \\
Field of Interest $\mathcal{A}_{0}$ & $\leftrightarrow$ & Set $S_{0}$ \\
Sensing area $\mathcal{A}_{i}$ of perimeter $L_{i}$ & $\leftrightarrow$ & Set $S_{i}$ of perimeter $L_{i}$ \\
Random sensor deployment & $\leftrightarrow$ & Random set placement \\
Trajectory of target X & $\leftrightarrow$ & Random line $\ell$ crossing $S_{0}$ \\
\hline Probability of target detection & $\leftrightarrow$ & Probability of $\ell$ intersecting \\
by at least $k$ sensors $P_{D}(k)$ & & at least $k$ sets \\
\hline
\end{tabular}

intersects at least $k$ out of the $N$ sets $S_{i}, i=1 \ldots N$.

Table I summarizes the mapping from the mobile target detection problem to the line-set intersection problem. Throughout the rest of the paper the terms sensing area $\mathcal{A}_{i}$ and set $S_{i}$ will be used interchangeably.

\section{Relevant Background}

To evaluate the detection probability $P_{D}$ using the line-set intersection formulation, we need to quantify the number of lines that intersect with the any of the sets $\mathcal{A}_{i}$, as well as the number of lines that intersect with the $F o I$. However, the set of lines in the plane intersecting a set $\mathcal{A}$ is uncountable. To bypass our difficulty in counting lines in the plane, we adopt a measure from Integral Geometry and Geometric Probability [14], [15]. In geometric probability, the measure $m(\ell)$ of a set of lines $\ell(\xi, \theta)$ in the plane is defined as follows [14], [15]:

Definition 1: Measure of set of lines $m(\ell)$ : The measure $m$ of a set of lines $\ell(\xi, \theta)$ is defined as the integral over the line density $d \ell=d \xi \wedge d \theta$

$$
m(\ell)=\int d \xi \wedge d \theta
$$

where $\wedge$ denotes the exterior product used in Exterior Calculus [17].

In the case where $\mathcal{A}$ is convex, the measure of the set of lines that intersect $\mathcal{A}$ is equal to:

$$
m(\ell: \ell \bigcap \mathcal{A} \neq \emptyset)=\int_{\ell \bigcap \mathcal{A} \neq \emptyset} d \xi \wedge d \theta=\int_{0}^{2 \pi} \xi d \theta=L
$$

where $L$ is the perimeter of $\mathcal{A}$. Interested reader is referred to [14], [15], for the proof of (2). In the case where $\mathcal{A}$ is nonconvex, the measure in (2) can be computed by observing that any line intersecting the convex hull of $\mathcal{A}$, also intersects $\mathcal{A}$. Hence, the measure of the set of lines that intersect a nonconvex set is equal to the perimeter of the convex hull of that set, denoted as $L^{h}$.

A geometric interpretation for (2), can be obtained by considering the thickness $T(\theta)$ of a bounded set $\mathcal{A}$ [15]:

Definition 2: Thickness of a bounded set $T(\theta)$ : The thickness of a bounded set $\mathcal{A}$ at direction $\theta$ is defined as the length of the projection of $\mathcal{A}$ to a line of direction $\theta$.

The thickness of a set $\mathcal{A}$ measures the set of lines along the direction perpendicular to $\theta$, that intersect $\mathcal{A}$. Figure 2(a), illustrates the thickness of a set $\mathcal{A}_{i}$ at direction $\theta$. Figure 2(b) 


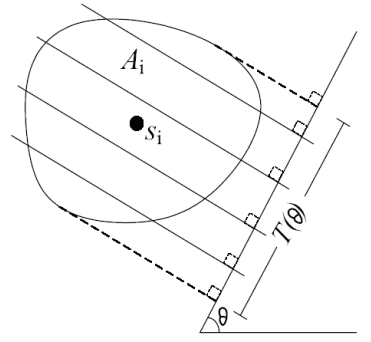

(a)

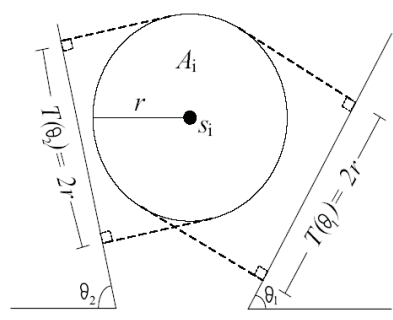

(b)
Fig. 2. (a) The thickness $T(\theta)$ of a set $\mathcal{A}_{i}$ is equal to the length of the projection of $\mathcal{A}_{i}$ on a line with direction $\theta . T(\theta)$ measures the set of lines of direction perpendicular to $\theta$ that intersect $\mathcal{A}_{i}$. (b) For the case of a disk, $T(\theta)=2 r, \forall \theta$, where $r$ is the radius of the disk $\mathcal{A}_{i}$.

illustrates the thickness of a circular sensing area $\mathcal{A}_{i}$, of radius $r$. Independent of the direction of projection, the thickness of a disk is always equal to the diameter of the sensing area, that is $T(\theta)=2 r, \forall \theta$. Thickness is related to $m(\ell)$ via:

$$
m(\ell)=\int_{\ell \cap \mathcal{A} \neq \emptyset} d \xi \wedge d \theta \stackrel{(\mathrm{i})}{=} \int_{0}^{\pi} T(\theta) d \theta \stackrel{(\mathrm{ii})}{=} \pi E(T)=L .
$$

Step (i) holds due to the fact that for a fixed $\theta$, the integral of $d \xi$ (set of positions) of the lines that intersect $\mathcal{A}$ is equal to $T(\theta)$. Step (ii) holds due to the uniform distribution of the lines:

$$
E(T)=\int_{0}^{\pi} \frac{1}{\pi} T(\theta) d \theta
$$

The relation between $m(\ell)$ and $L$ as expressed in (3) can be interpreted as follows. The measure $m(\ell)$ of the set of lines $\ell(\xi, \theta)$ intersecting a bounded set $\mathcal{A}$ is equal to the average length $E(T)$ of the projection of $\mathcal{A}$ over all possible directions, times the measure of all the possible directions.

\section{Target Detection Probability}

In this section, we analytically evaluate the detection probability $P_{D}(k)$, that a target crossing the $F o I$ is detected by at least $k$ sensors. We then evaluate $P_{D}(k)$ under the SD model. Finally, we compute the mean time until a target $X$ crossing the $F o I$ is first detected.

\section{A. Instant Detection}

Under the ID model, the probability that target $X$ is detected by at least $k$ sensors is given by the following theorem.

Theorem 1: Let $\mathcal{A}_{0}$ be a bounded FoI of perimeter length $L_{0}$ monitored by $N$ sensors randomly deployed within $\mathcal{A}_{0}$, with sensor $s_{i}, i=1 \ldots N$ having a sensing area of perimeter length $L_{i}$. The probability $P_{D}(k)$ that at least $k \geq 1$ sensors detect a target $X$ crossing the FoI and moving on a straight line is given by:

$$
P_{D}(k)=1-\sum_{w=0}^{k-1} \sum_{j=1}^{\left|Z_{N, w}\right|} \prod_{i=1}^{\left|z_{j}\right|} q_{z_{j}(i)} \prod_{v=1}^{|\bar{z} j|}\left(1-q_{\bar{z}_{j}(v)}\right),
$$

where $Z_{N, w}$ denotes the $\left(\begin{array}{l}N \\ w\end{array}\right) w$-tuples $z_{j}$ of vector $[1, \ldots, N]$. That is, $Z_{N, w}=\left\{z_{j}: z_{j(1)}, \ldots, z_{j(i)}, \ldots, z_{j(w)} \mid j(i) \in\right.$
$[1, N], j(i) \neq j(g), \forall i \neq g\}$. The $\bar{z}_{j}$ denotes the complement $(N-w)$-tuple of $z_{j}$ with respect to vector $[1, \ldots, N]$, and $q_{i}$ is given by $q_{i}=\frac{L_{i}}{L_{0}}$.

Proof: Let us first compute the probability that a target is detected by a single sensor $s_{i}$. Based on our mapping in Section III-B, this event is equivalent to the conditional probability $q_{i}$ that a line intersecting $\mathcal{A}_{0}$, also intersects $\mathcal{A}_{i}$. This probability is equal to the quotient of the measure of the set of lines that intersect both $\mathcal{A}_{0}, \mathcal{A}_{i}$ over the measure of the set of lines that intersect $\mathcal{A}_{0}$.

$$
q_{i}=\frac{m\left(\ell \bigcap \mathcal{A}_{0} \bigcap \mathcal{A}_{i} \neq \emptyset\right)}{m\left(\ell \bigcap \mathcal{A}_{0} \neq \emptyset\right)} \stackrel{\text { (i) }}{=} \frac{m\left(\ell \bigcap \mathcal{A}_{i} \neq \emptyset\right)}{m\left(\ell \bigcap \mathcal{A}_{0} \neq \emptyset\right)} \stackrel{\text { (ii) }}{=} \frac{L_{i}}{L_{0}} .
$$

Step (i) holds due to the fact that $\mathcal{A}_{i}$ is within $\mathcal{A}_{0}$ and hence, any line intersecting $\mathcal{A}_{i}$ also intersects $\mathcal{A}_{0}$. Step (ii) follows due to (2). The probability $q_{i}$ in (6) is computed for the case where both $\mathcal{A}_{0}, \mathcal{A}_{i}$ are convex sets. In the case where any of the sets are not convex, the length of the perimeter of the convex hull, denoted as $L^{h}$, is used to compute $q_{i}$.

Using (6), we now compute the probability that a line $\ell$ intersects exactly $k$ sets. Let $Z_{N, k}$ denote the $\left(\begin{array}{c}N \\ k\end{array}\right) k$ tuples $z_{j}$ of vector $[1, \ldots, N]$. That is, $Z_{N, k}=\left\{z_{j}\right.$ : $\left.z_{j(1)}, \ldots, z_{j(i)}, \ldots, z_{j(k)} \mid j(i) \in[1, N], j(i) \neq j(g), \forall i \neq g\right\}$. Let also $\bar{z}_{j}$ denote the complement of $z_{j}$ with respect to the vector $[1, \ldots, N]$. The probability that a line $\ell$ intersects all sets indicated by the $k$-tuple $z_{j}$ is given by:

$$
\begin{aligned}
P\left(z_{j}\right) \stackrel{\text { (i) }}{=} & P\left(\ell \bigcap \mathcal{A}_{z_{j(1)}} \neq \emptyset, \ldots, \ell \bigcap \mathcal{A}_{z_{j(k)}} \neq \emptyset\right. \\
& \left.\ell \bigcap \mathcal{A}_{\bar{z}_{j(1)}}=\emptyset, \ldots, \ell \bigcap \mathcal{A}_{\bar{z}_{j(N-k)}}=\emptyset\right) \\
\stackrel{\text { (ii) }}{=} & P\left(\ell \bigcap \mathcal{A}_{z_{j(1)}} \neq \emptyset\right) \ldots P\left(\ell \bigcap \mathcal{A}_{z_{j(k)}} \neq \emptyset\right) \\
& P\left(\ell \bigcap \mathcal{A}_{\bar{z}_{j(1)}}=\emptyset\right) P\left(\ldots \ell \bigcap \mathcal{A}_{\bar{z}_{j(N-k)}}=\emptyset\right) \\
= & \prod_{i=1}^{\left|z_{j}\right|} q_{z_{j}(i)} \prod_{v=1}^{|\bar{z} j|}\left(1-q_{\bar{z}_{j}(v)}\right),
\end{aligned}
$$

In step (i), we express $P\left(z_{j}\right)$ as the probability that a random line intersects exactly the $k$ sets denoted by the $k$-tuple $z_{j}$. Since the sets $\mathcal{A}_{i}$ are randomly and independently deployed within the FoI, in step (ii) the probability of the intersection of events becomes equal to the product of the probabilities of the individual events. To compute the probability of a random line intersecting any $k$ sets, $P\left(z_{j}\right)$ must be summed over all possible $k$-tuples $z_{j}$.

$$
P\left(Z_{N, k}\right)=\sum_{Z_{N, k}} \prod_{i=1}^{\left|z_{j}\right|} q_{z_{j}(i)} \prod_{v=1}^{|\bar{z} j|}\left(1-q_{\bar{z}_{j}(v)}\right) .
$$

Theorem 1 holds by noting that

$$
P_{D}(k)=1-\sum_{w=0}^{k-1} P\left(Z_{N, w}\right) .
$$

From Theorem 1, note that $P_{D}(k)$ depends only on the ratios $\frac{L_{i}}{L_{0}}$ of $\mathcal{A}_{i}$ and not the specific shape, or size of 
the sensing areas. Hence, Theorem 1 allows the analytic computation of the detection probability in the case of sensors with heterogeneous sensing areas. However, the complexity of computing (5) grows exponentially with the heterogeneity of the sensing areas. For large-scale networks, $P_{D}(k)$ can be efficiently computed with the use of the following result.

Theorem 2: Let the probability $q_{i}$ that a target $X$ is detected by sensor $s_{i}$ be small, while the sum of the probabilities $\sum_{i} q_{i}$ is nearly a constant $\gamma$, as $i \rightarrow \infty$. The probability $P\left(Z_{N, k}\right)$ converges to a Poisson distribution of rate $\gamma$.

$$
P\left(Z_{N, k}\right)=\frac{\gamma^{k}}{k !} e^{-\gamma}, \quad \sum_{i} q_{i} \rightarrow \gamma, \quad \max _{i} q_{i} \rightarrow 0 .
$$

Proof: The proof of Theorem 2 is a special case of Lindeberg's Central Limit Theorem and is provided in [18].

Substituting (10) to (9) yields $P_{D}(k)$, for the case of largescale heterogeneous $\mathrm{SN}$. If sensors have sensing areas with perimeters of equal length (not necessarily identical shapes), (5) can be simplified to the following form.

Corollary 1: The probability $P_{D}(k)$ that a target crossing the $F o I$ will be detected by at least $k$ sensors, when all sensors have sensing areas with equal perimeters $L$ is equal to:

$$
P_{D}(k)=1-\sum_{i=0}^{k-1}\left(\begin{array}{c}
N \\
i
\end{array}\right) \frac{L^{i}\left(L_{0}-L\right)^{N-i}}{L_{0}^{N}} .
$$

Proof: Corollary 1 follows by setting $q_{i}=\frac{L}{L_{0}}$ in (5).

Using Theorem 1, we can also evaluate the probability that a target $X$ remains undetected by any sensor. Corollary 2 , computes the probability $P_{M}$ of missing a target.

Corollary 2: The probability $P_{M}$ that a target crossing the FoI is not detected by any sensor is equal to:

$$
P_{M}=\prod_{i=1}^{N}\left(1-\frac{L_{i}}{L_{0}}\right) .
$$

Proof: The proof of Corollary 2 follows, by observing that $P_{M}=P\left(Z_{N, 0}\right)$, and $z_{j}=\emptyset, \bar{z}_{j}=\{1, \ldots, N\}$.

Depending on the application, (12) allows us to select $L_{i}, N$ so that $P_{M}$ remains below any desired threshold value.

\section{B. Sampling Detection}

Under the SD model, a target $X$ must remain within the sensing area of a sensor $s_{i}$ for at least $t_{t h}$ units of time, before $s_{i}$ can detect $X$. Given a velocity model for $X$, SD occurs if the length of the intersection of the trajectory of $X$ with the sensing area $\mathcal{A}_{i}$ is at least $l_{t} h(v)$. Assuming the simplest velocity model of constant speed ${ }^{1}$ for $X$, SD occurs when the length of the intersection is longer than $v t_{t h}$. To measure the set of lines that intersect a set $\mathcal{A}$ and have a cord within $\mathcal{A}$ of length longer than $v t_{t h}$ we define the notion of effective thickness $T^{\prime}(\theta)$.

Definition 3: Effective Thickness $T^{\prime}(\theta)$ : The effective thickness $T^{\prime}(\theta)$ for a set $\mathcal{A}$ is defined as the measure of the

\footnotetext{
${ }^{1}$ Any other velocity model can be assumed to map the sampling time threshold $t_{t h}$ into a trajectory length $l_{t h}(v)$.
}

set of lines $m(\ell)$ perpendicular to the direction $\theta$, for which the length of the cord within $\mathcal{A}$ is greater or equal $v t_{t h}$. That is,

$$
T^{\prime}(\theta)=\int_{|\ell(\xi, \theta) \cap \mathcal{A}| \geq v t_{t h}} d \xi
$$

The probability of moving target detection under the SD model is given by the following theorem.

Theorem 3: Let a target $X$ cross the FoI moving on a straight line at a constant speed $v$. The probability $P_{D}(k)$ that at least $k$ sensors detect $X$ when the target must be sensed for at least time $t_{t h}$ is given by:

$$
P_{D}(k)=1-\sum_{w=0}^{k-1} \sum_{Z_{N, w}} \prod_{i=1}^{\left|z_{j}\right|} q_{z_{j}(i)}^{\prime} \prod_{v=1}^{|\bar{z} j|}\left(1-q_{\bar{z}_{j}(v)}^{\prime}\right),
$$

where $q_{i}^{\prime}=\frac{E\left(T_{i}^{\prime}\right)}{E\left(T_{0}\right)}$.

Proof: The proof of Theorem 3, follows the same steps of the proof of Theorem 1 in the case of the ID model. The only difference between the two proofs is the computation of the probability $q_{i}$ for a single sensor to detect a target $X$. Based on our mapping in Section III-B, in the case of the $\mathrm{SD}$ model, target detection is equivalent to the conditional probability $q_{i}^{\prime}$ that a line that intersects $\mathcal{A}_{0}$, also intersects $\mathcal{A}_{i}$, with the length of the cord being $\left|\ell \bigcap \mathcal{A}_{i}\right| \geq v t_{t h}$. This probability is equal to the quotient of the measure of the lines that intersect both $\mathcal{A}_{0}, \mathcal{A}_{i}$ and have a cord length $\left|\ell \bigcap \mathcal{A}_{i}\right| \geq$ $v t_{t h}$, over the measure of the set of lines that intersect $\mathcal{A}_{0}$.

$$
q_{i}^{\prime}=\frac{m\left(\left|\ell \bigcap \mathcal{A}_{0} \bigcap \mathcal{A}_{i}\right| \geq v t_{t h}\right)}{m\left(\ell \bigcap \mathcal{A}_{0} \neq \emptyset\right)}
$$

The measure of the set of lines that intersect $\mathcal{A}_{0}$ is given by (2) and is equal to $E\left(T_{0}\right)=L_{0}$. The measure of the set of lines that intersect both $\mathcal{A}_{0}, \mathcal{A}_{i}$ and have a cord length $\left|\ell \bigcap \mathcal{A}_{i}\right| \geq v t_{t h}$, is computed as follows:

$$
\begin{aligned}
m\left(\left|l \bigcap \mathcal{A}_{0} \bigcap \mathcal{A}_{i}\right| \geq v t_{t h}\right) & \stackrel{\text { (i) }}{=} m\left(\left|\ell \bigcap \mathcal{A}_{i}\right| \geq v t_{t h}\right) \\
& \stackrel{\text { (ii) }}{=} \int_{\left|\ell \bigcap \mathcal{A}_{i}\right| \geq v t_{t h}} d \xi \wedge d \theta \\
& \stackrel{\text { (iii) }}{=} \int_{0}^{\pi} T_{i}^{\prime}(\theta) d \theta \stackrel{\text { (iv) }}{=} \pi E\left(T^{\prime}\right) .
\end{aligned}
$$

In step (i), $\mathcal{A}_{i}$ is a subset of $\mathcal{A}_{0}$ and, hence, the length of the line that is common to both $\mathcal{A}_{0}$ and $\mathcal{A}_{i}$ is equal to the length of the cord in $\mathcal{A}_{i}$. In step (ii), we integrate the line density $d l=d \xi \wedge d \theta$ over all lines that intersect $\mathcal{A}_{i}$ and have a length of at least $v t_{t h}$. In step (iii), for a fixed direction $\theta$ the integral of $d \xi$ over all lines for which $\left|\ell \bigcap \mathcal{A}_{i} \geq v t_{t h}\right|$ is equal to the effective thickness $T^{\prime}(\theta)$. The average effective thickness for random lines is given by:

$$
E\left(T^{\prime}\right)=\int_{0}^{\pi} \frac{1}{\pi} T^{\prime}(\theta) d \theta .
$$

Hence, step (iv) follows. The combination of (15), (16), (2) and (3) yields:

$$
q_{i}^{\prime}=\frac{\pi E\left(T_{i}^{\prime}\right)}{L_{0}}=\frac{\pi E\left(T_{i}^{\prime}\right)}{\pi E\left(T_{0}\right)}=\frac{E\left(T_{i}^{\prime}\right)}{E\left(T_{0}\right)}
$$




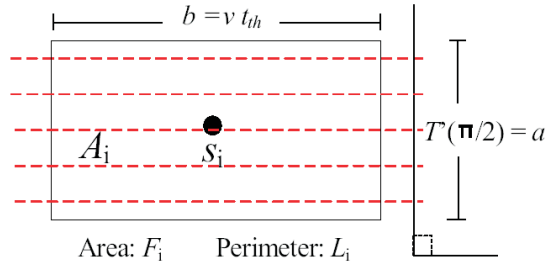

(a)

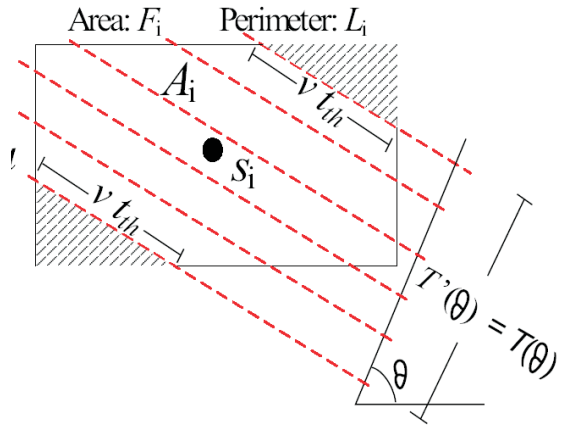

(b)

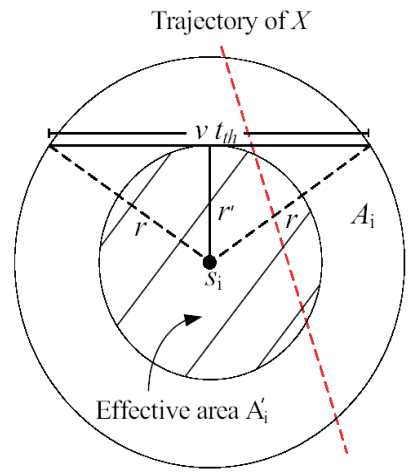

(c)

Fig. 3. (a) The effective thickness of a rectangle on direction $\theta=0$, (b) the effective thickness of a rectangle on a random direction $\theta$, (c) the effective sensing area of a disk.

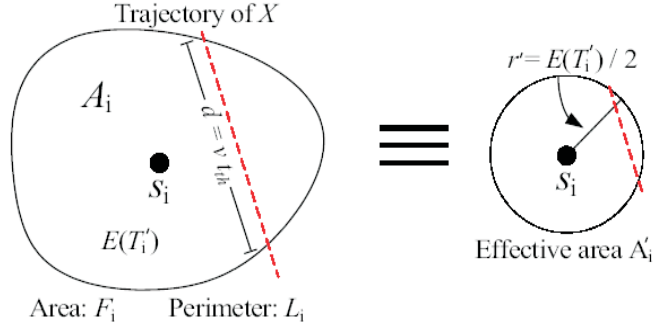

Fig. 4. The equivalent effective area for a sensor $s_{i}$.

Following the same steps as in the proof of Theorem 1, yields Theorem 3.

\section{Mapping the SD Model to the ID Model}

The ID model facilitates a geometric interpretation of the target detection problem. Any target crossing the sensing area of a sensor is detected. However, no such geometric interpretation exists for the SD model. We now provide a reduction from the SD model to the ID model that allows us to map any results for the simpler ID model to the SD one. Our goal is to define for each sensor $s_{i}$, an effective sensing area $\mathcal{A}_{i}^{\prime}$ with the following property. If a target $X$ crosses the boundary of $\mathcal{A}_{i}^{\prime}$ (ID model), then $X$ is detected under the SD model.

For sensing areas of arbitrary shape, the average effective thickness of $\mathcal{A}_{i}$, does not correspond to the average thickness of a subset of $\mathcal{A}_{i}$. As an example, in figure 3(a), all lines of direction $\frac{\pi}{2}$ intersecting the rectangular sensing area $\mathcal{A}_{i}$, have a line segment within $\mathcal{A}$ longer than $v t_{t h}$ (assuming $v t_{t h} \leq b$.). However, for a direction $\theta \neq\left\{0, \frac{\pi}{2}, \pi\right\}$ there is a set of lines with a line segment within $\mathcal{A}_{i}$ shorter than $v t_{t h}$. The subset of $\mathcal{A}_{i}$ that does not result in detection for lines in direction $\theta$ is depicted by the shaded areas in figure $3(\mathrm{~b})$. Hence, one cannot define a subset of $\mathcal{A}_{i}$ with average thickness equal to the average effective thickness of $\mathcal{A}_{i}$.

However, from (14), the probability of detection $P_{D}(k)$ only depends on $E\left(T_{i}^{\prime}\right)$, and not the shape of the sensing area. Hence, we can define an effective sensing area $\mathcal{A}^{\prime}$ for each sensor $s_{i}$, that is not necessarily a subset of $\mathcal{A}$.
Definition 4: Effective Sensing Area $\mathcal{A}^{\prime}$ : Let the average effective thickness of a set $\mathcal{A}$ be equal to $E\left(T^{\prime}\right)$. The effective sensing area $\mathcal{A}^{\prime}$ is defined as a disk of radius $r^{\prime}=E\left(T^{\prime}\right) / 2$.

Using the notion of the effective sensing area, we can map the target detection probability under the SD model, to a target detection problem under the ID model using the following corollary.

Corollary 3: The target detection probability under the SD model is equal to the target detection probability under the ID model, when the sensing areas of the sensors are replaced by the effective sensing areas.

Proof: The proof follows by setting $L_{i}=2 \pi r^{\prime}$ in (5).

In figure 4 , we show the equivalence between the sensing area of a sensor $s_{i}$ under the SD model. Note that in the case of the unit disk model, the effective sensing area is a subset of the original sensing area. As an example, in figure 3(c) the effective sensing area of a disk of radius $r$, is a concentric disk of radius $r^{\prime}=\sqrt{r^{2}-\left(\frac{v t_{t h}}{2}\right)^{2}}$. Through the rest of the paper we focus on the ID model, with equivalent results holding for the SD model.

\section{Mean Free Path until the First Detection}

In several applications, the distance that the target $X$ travels within the FoI undetected is an important metric of the quality of detection. In this section, we analytically compute the mean free path $E(\sigma)$ until the first detection of a target $X$. To facilitate the computation we assume that sensors have identical sensing areas. Note that computing the mean free path $E(\sigma)$ traveled by $X$, is sufficient to determine the mean time $E(t)$ until $X$ is first detected, given a velocity model for $X$. The mean free path problem can be stated as follows.

Mean Free Path Problem: Let $N$ sensors with identical sensing areas be randomly and independently deployed within a $F o I$ of area $F_{0}$. Assuming that a target $X$ is moving on a straight line, compute the mean free path before the target $X$ is detected.

The mean free path for which the target $X$ remains undetected is given by the following theorem.

Theorem 4: Let $N$ sensors with identical sensing areas be randomly and independently deployed within a FoI of area 


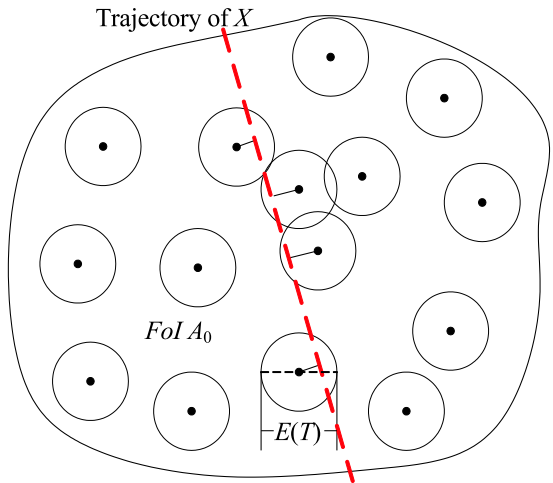

(a)

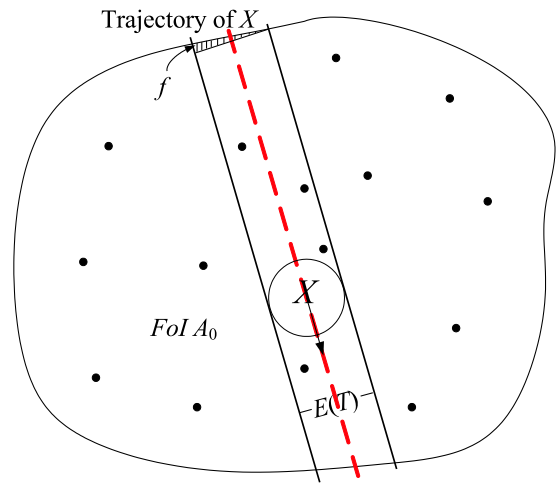

(b)

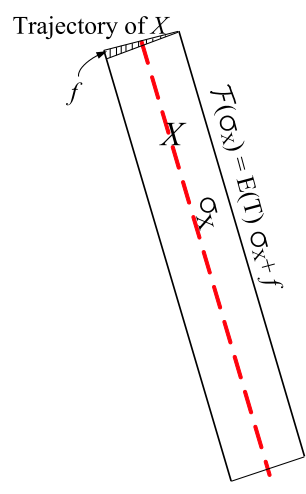

(c)

Fig. 5. (a) Any sensor within a distance $\frac{E(T)}{2}$ from the trajectory of the target $X$, detects $X$, (b) Equivalent formulation, for a target of average thickness $E(T)$, and sensors with sensing areas reduced to point masses, detection occurs if a sensor $s_{i}$ "collides" with the target, (c) the mean free path of a target $X$ and the equivalent free area.

$F_{0}$. Assuming a target $X$ moving on a straight line, the mean free path before $X$ is detected equals:

$$
E(\sigma) \approx \frac{F_{0}}{N E(T)}
$$

Proof: Under the ID model, a target $X$ travels for a distance $\sigma_{X}$ undetected, if it does not cross the sensing area of any sensor. When the sensors have a sensing area of identical thickness for all $\theta$, on a given trajectory, any sensor within distance $\frac{E(T)}{2}$ from the trajectory of $X$, detects $X$. This event is equivalent to considering that the target $X$ has an average thickness of $E(T)=T(\theta), \forall \theta$, while the sensing area of all sensors is reduced to point masses. Figure 5(a) illustrates target $X$ being detected by sensors with sensing areas of average thickness $E(T)$ while it crosses the $F o I$. Figure 5(b) illustrates the equivalent scenario, where the target $X$ has an average thickness of $E(T)$, while the sensing areas of the sensors are reduced to point masses.

When the target $X$ moves a distance $\sigma_{X}$, it covers an area of size $\mathcal{F}\left(\sigma_{X}\right)=E(T) \sigma_{X}+f$, where $f$ denotes the residual shaded area in figure $5(\mathrm{c})$. Hence, the probability that the target $X$ is not detected for a distance of $\sigma \geq \sigma_{X}$ is equal to the probability that no sensor is located within $\mathcal{F}\left(\sigma_{X}\right)$. Given that the sensors are randomly and independently deployed, the number of sensors within $\mathcal{F}\left(\sigma_{X}\right)$ is given by a homogeneous planar Poisson point process of density $\rho=\frac{N}{F_{0}}[14]$ :

$$
P(|S|=k)=\frac{(\rho F)^{k}}{k !} e^{-\rho F}
$$

where $|S|$ denotes number of sensors. Equation (19) holds under the assumption that $F_{0} \rightarrow \infty$, while the sensor density $\rho$ remains constant. Based on (19), the probability that the free path of target $X$ is $\sigma \geq \sigma_{X}$, is equal to the probability that no sensors exist within an area of size $\mathcal{F}\left(\sigma_{X}\right)$ :

$$
\begin{aligned}
P\left(\sigma \geq \sigma_{X}\right) & =P\left(N_{\left.\mathcal{F}(\sigma)_{X}\right)}=0\right) \\
& =e^{-\rho \mathcal{F}(\sigma)}=e^{-\rho(E(T) \sigma+f)} .
\end{aligned}
$$

The random variable $\sigma$ is a non-negative continuous random value and, hence its expectation is given by:

$$
E(\sigma)=\int_{0}^{Q} P\left(\sigma \geq \sigma_{X}\right) d \sigma_{X}=\frac{e^{-\rho f}}{\rho E(T)}\left(1-e^{-\rho E(T) Q}\right),
$$

where $Q$ denotes the maximum possible length of the trajectory of $X$ within the FoI. When the residual area $f$ is small enough so that $e^{-\rho f} \approx 1$ and $Q$ is long enough so that $e^{-\rho E(T) Q} \approx 0$,

$$
E(\sigma) \approx \frac{1}{\rho E(T)}=\frac{F_{0}}{N E(T)}
$$

Note that in the present analysis we have assumed sensing areas of constant thickness $T(\theta), \forall \theta$. This assumption can by relaxed if one assumes sensing areas of equal thickness on a given direction $\theta$, but not constant over all $\theta$, and then average over all $\theta$.

\section{VAlidation of the Theoretical Results}

In this section, we verify the validity of our theoretical results be performing extensive simulations. We randomly deployed $N$ sensors in a circular $F_{O} I$ of radius $R=100 \mathrm{~m}$. We then generated random lines corresponding to random trajectories of targets and measured the number of sensors that detect the moving targets. We performed the following experiments.

\section{A. Probability of Detection by a Single Sensor}

In our first experiment,we randomly deployed a single sensor, with a circular sensing area of radius $r$. We varied $r$ from $10 \mathrm{~m}$ to $80 \mathrm{~m}$ and measured the probability that a target moving at a random trajectory is detected by the sensor. For each radius $r$ we repeated the experiment 100 times to ensure statistical validity. Based on our derivations in Section IV, the probability that the target is detected is equal to:

$$
P_{D}=q_{i}=\frac{L_{i}}{L_{0}}=\frac{r}{R}
$$

In figure 6(a) we show the probability of detection $P_{D}$ for varying $r$ for our first experiment. We observe an almost exact 


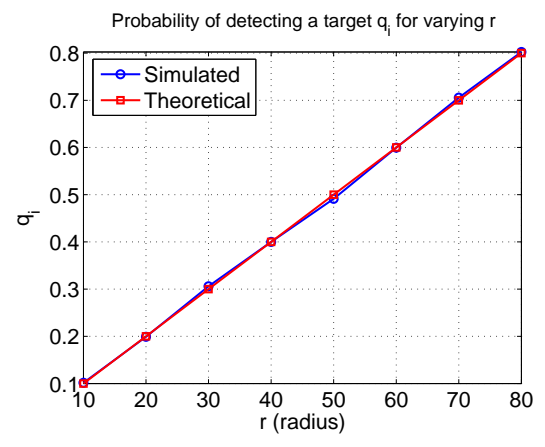

(a)

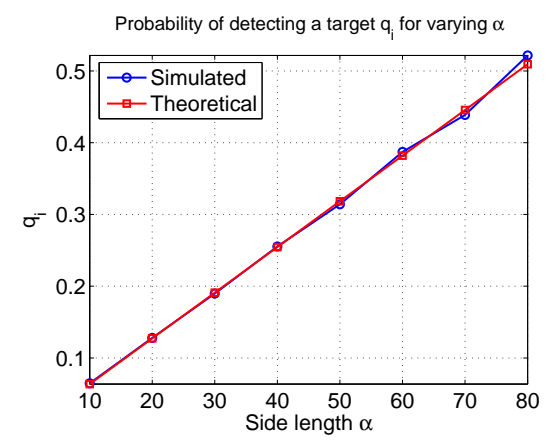

(b)

Fig. 6. Homogeneous SN: (a) Probability of detecting a target by the deployment of a single sensor with circular sensing area, as a function of the radius $r$. (b) Probability of detecting a target by the deployment of a single sensor with square sensing area, as a function of the side $\alpha$.

match between the theory and the simulation, confirming that the probability of a random line intersecting a set of perimeter $L_{i}$ given that it intersects the overset of perimeter $L_{0}$ is equal to the quotient of the two perimeters.

We also repeated our experiment when the deployed sensor had a square sensing area of perimeter $4 * \alpha$, where $\alpha$ denotes the length of the side of the square and was varied from $10 \mathrm{~m}$ to $80 \mathrm{~m}$. In figure $6(\mathrm{~b})$ we show the probability of detection $P_{D}$ for varying $\alpha$ for the case of square sensing area. We observe that regardless of the shape of the sensing area, our theoretical formula agrees with the simulation.

\section{B. Probability of Detection in Heterogeneous SN}

In our second experiment we deployed sensors with heterogeneous sensing capabilities and evaluated the detection performance of the SN. Each sensor deployed had circular sensing area of a radius uniformly distributed in $[0,1]$. We selected a small sensing area in order to satisfy the condition $\max _{i} q_{i} \rightarrow 0$ while $\sum_{i} q_{i} \rightarrow \gamma$, so that the probability of detection of a target by exactly $k$ sensors can be approximated by (10). We varied the number of sensors deployed from $N=100$ to $N=1000$, and computed $P_{D}(1)$. The exact formula for $P_{D}(1)$ is given by

$$
P_{D}(1)=\prod_{i=1}^{N}\left(1-\frac{L_{i}}{L_{0}}\right)
$$

For large $N$ according to Theorem $2, P_{D}(1)$ tends to

$$
P_{D}(1)=1-e^{-\sum_{i=1}^{N} \frac{L_{i}}{L_{0}}} .
$$

In figure 7(a), we show the theoretical value of $P_{D}(1)$, the value according to Theorem 2 , and the simulated value, as a function of $N$. We observe that when the conditions of Theorem 2 are satisfied, one can compute $P_{D}(k)$ without incurring the high computational cost of the exact formula (as $k$ increases the number of terms in the exact formula of $P_{D}(k)$ increase exponentially).

In figure $7(\mathrm{~b})$, we show $P_{D}(1)$ when the radius of the sensors is uniformly distributed in $[0,0.1]$. We observe that the target detection probability grows almost linearly with the number of sensors is deployed when the length of the perimeters of the sensing areas of the devices deployed are significantly smaller than the perimeter of the FoI. We also observe that the Poisson approximation is very close to the exact theoretical value as well as the simulated value.

\section{Probability of Detection in Homogeneous SN}

In our third experiment, we evaluated the detection performance of a SN when all deployed sensors have identical sensing areas. We initially deployed 30 sensors with a circular sensing area of radius $r=10 \mathrm{~m}$ and measured the probability of detection $P\left(Z_{N_{k}}\right)$ that a target randomly crossing the $F o I$ is detected by exactly $k$ sensors. The probability $P\left(Z_{N, k}\right)$ for the homogeneous case is given by:

$$
P\left(Z_{N, k}\right)=\left(\begin{array}{c}
N \\
k
\end{array}\right)\left(\frac{L}{L_{0}}\right)^{k}\left(1-\frac{L}{L_{0}}\right)^{N-k} .
$$

In figure 7(c) we show $P\left(Z_{N, k}\right)$ for a homogeneous $\mathrm{SN}$ as a function of $k$. We also evaluate the probability $P_{D}(k)$ that a target would be detected by at least $k$ sensors, that in the homogeneous case is given by (11). In figure 7(d), we show $P_{D}(k)$ as a function of $k$. We observe that our theoretical formulas match the simulation results.

We also evaluated the probability $P_{M}$ of not detecting a target crossing the sensor field as well as the probability of detection by at least one sensor $P_{D}(1)$, a function of the number of sensors deployed. In figure 6(e) we show $P_{M}$ as a function of $N$. In figure 7(f) we show $P_{D}(1)$ as a function of $N$. From figures 7(e), 7(f), one can select $N$ so that the $P_{D}(1)$ is above a threshold. As an example, if $P_{D}(1) \geq 95 \%$ more than 30 sensors must be deployed.

Figure 7(f), also shows the fraction of the FoI denoted as $\operatorname{Fr}\left(\mathcal{A}_{0}\right)$ covered by at least one sensor. From figure 7(f), we note that it is not necessary to cover the entire FoI to achieve detection probability close to unity. Thus, target mobility helps detecting targets with a significantly smaller number of sensors, compared to the number required for detecting static targets.

\section{CONCLUSION}

We studied the problem of quantifying the target detection capability of heterogeneous SN. We mapped the mobile target detection problem to the line-set intersection problem and derived analytical expressions for the probability of target 


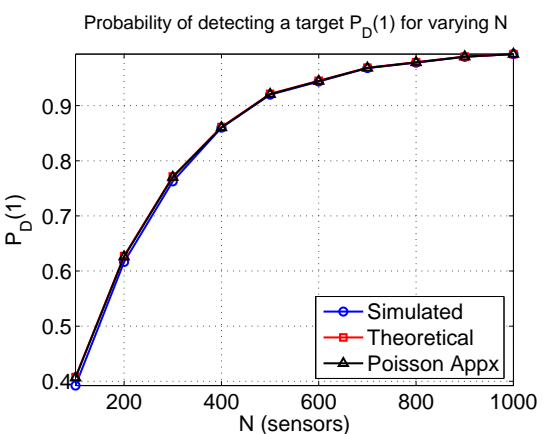

(a)

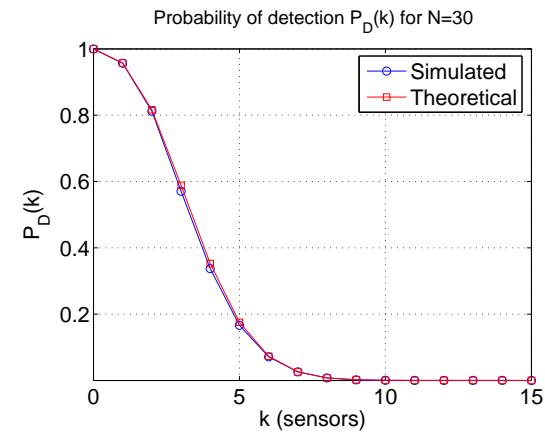

(d)

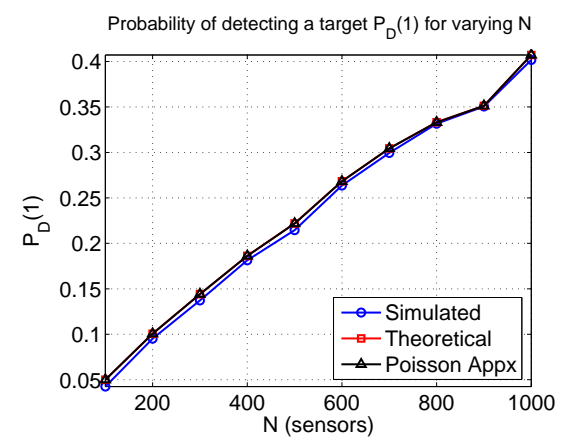

(b)

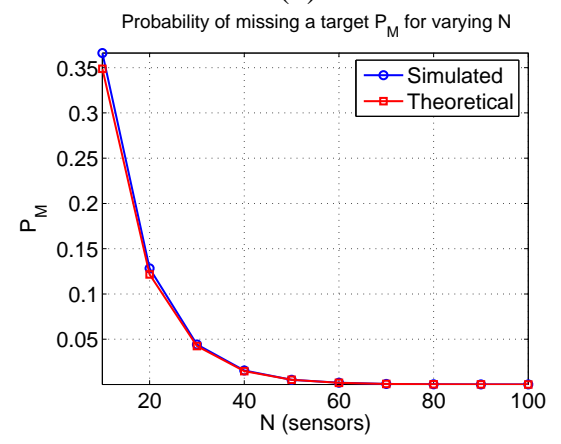

(e)

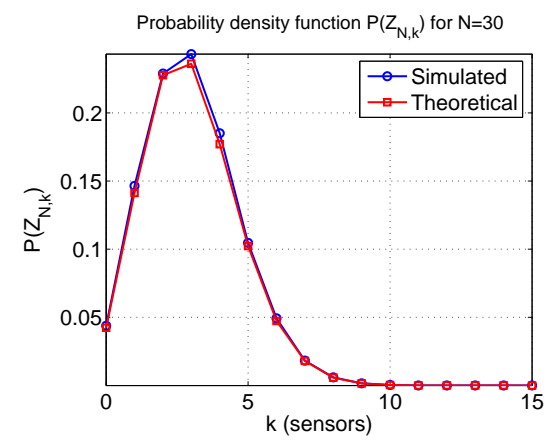

(c)

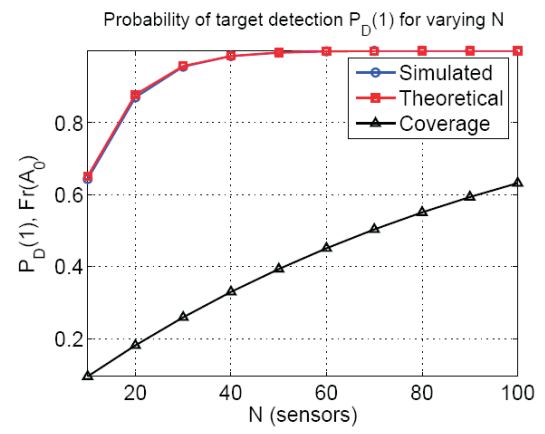

(f)

Fig. 7. (a) Heterogeneous SN: Probability of target detection by at least one sensor as a function of the network size, when the radius of the sensing area is uniformly distributed within $r \in[0,1]$. (b) Probability of target detection by at least one sensor as a function of the network size, when the radius of the sensing area is uniformly distributed within $r \in[0,0.1]$. (c) Homogeneous SN: Target detection probability by exactly $k$ sensors. (d) Target detection probability by at least $k$ sensors. (e) Probability of missing a target as a function of the network size. (f) Probability of target detection by at least one sensor as a function of the network size, and comparison with the fraction of the FoI covered by at least one sensor.

detection in stochastically deployed heterogeneous SN. We showed that the target detection probability depends only on the length of the perimeters of the sensing areas of the sensors and not on their shape or area. We also analytically evaluated the mean free path until a target is first detected, a critical measure of timely target detection. We verified our theoretical results via detailed simulations.

\section{REFERENCES}

[1] F. Koushanfar, S. Slijepcevic, M. Potkonjak and A. SangiovanniVincentelli, "Error-Tolerant Multi-Modal Sensor Fusion," In Proc. of IEEE CAS Workshop on Wireless Communication and Networking, 2002.

[2] C. Gui, and P. Mohapatra, "Power Conservation and Quality of Surveillance in Target Tracking Sensor Networks," In Proc. of the 10th Annual International Conference on Mobile Computing and Networking, Sept. 2004, pp. 129-143.

[3] Q. Cao, T. Yan, T. Abdelzaher, and J. Stankovic, "Analysis of Target Detection Performance for Wireless Sensor Networks," In Proc. of the International Conference on Distributed Computing in Sensor Networks, CA, June 2005.

[4] J. Aslam, Z. Butler, F. Constantin, V. Crespi, G. Cybenko, and D. Rus, "Tracking a Moving Object with a Binary Sensor Network," In Proc. of the 1st international Conference on Embedded Networked Sensor Systems, Nov. 2005, pp. 150-161.

[5] K. Chakrabarty, S. Iyengar, H. Qi and E. Cho, "Grid Coverage for Surveillance and Target Location in Distributed Sensor Networks," IEEE Transactions on Computers, vol. 51, pp. 1448-1453, December 2002.

[6] H. Yang, and B. Sikdar, "A Protocol for Tracking Mobile Targets using Sensor Networks," In Proc. of the IEEE Workshop on Sensor Network Protocols and Applications, May 2003, pp.7181.

[7] D. Li, K. Wong, Y. Hu, and A. Sayeed. "Detection, Classification and Tracking of Targets in Distributed Sensor Networks", IEEE Signal Processing Magazine, vol. 19 no. 2, March, 2002.
[8] A. Arora, P. Dutta, S. Bapat, V. Kulathumani, and H. Zhang, "A Line in the Sand: A Wireless Sensor Network for Target Detection, Classification, and Tracking," Computer Networks (Elsevier), vol. 46 no. 5 pp. 605-634, Dec. 2004.

[9] S. Meguerdichian, F. Koushanfar, G. Qu, and M. Potkonjak, "Exposure In Wireless Ad Hoc Sensor Networks," In Proc. of MOBICOM 2001, July 2001, pp. 139-150.

[10] S. Meguerdichian, F. Koushanfar, M. Potkonjak, and M. B. Srivastava, "Coverage Problems in Wireless Ad-Hoc Sensor Networks," in Proc. of INFOCOM, April 2001, pp. 1380-1387.

[11] T. Clouqueur, V. Phipatanasuphorn, P. Ramanathan, and K. K. Saluja, "Sensor Deployment Strategy for Target Detection", Proc. of the 1st ACM International Workshop on Wireless Sensor Networks and Applications, Sep. 2002, pp. 42-48.

[12] G. He, and J. Hou,"Tracking Targets with Quality in Wireless Sensor Networks," In Proc. of IEEE ICNP'05, November 2005.

[13] T. Clouqueur, K. K. Saluja, and P. Ramanathan, "Fault Tolerance in Collaborative Sensor Networks for Target Detection," IEEE Transaction on Computers, 2003.

[14] L. Santalo, Integral Geometry and Geometric Probability, AddisonWesley Publishing Company, 1976.i

[15] H. Solomon, Geometric Probability, CBMS-NSF, 1978.

[16] J. Sylvester, "On a Funicular Solution of Buffon's Needle Problem," Acta. Math. 14, 1890, pp. 185-205.

[17] H. Flanders, Differential Forms Prentice Hall, New Jersey, 1967.

[18] K. Itô, Introduction to Probability Theory, Cambridge University Press, 1984.

[19] C. Huang, and Y. Chen, "The Coverage Problem in Wireless Sensor Networks," In Proc. of the ACM International Workshop on Wireless Sensor Networks and Applications (WSNA), 2003.

[20] W. Feller, An Introduction to Probability Theory and its Aplications, 3rd edition, John Wiley and Sons Inc.

[21] S. Kumar, T. Lai, and A. Arora, "Barrier Coverage with wireless sensors," In Proc. of MOBICOM 2005, pp. 284-298. 